\title{
Cardiology
}

\section{Antiremodelling Efficacy and Clinical Safety of Zofenopril in Patients with Grade 1 and 2 Hypertension}

\author{
G. A. Khamidullaeva, PhD, ScD; G. J. Abdullaeva, PhD; L. Sh. Khafizova, PhD; \\ N. Z. Srojidinova*, PhD; N. B. Tursunova, PhD; D. R. Kurbanova, PhD, ScD; \\ N. Sh. Shakirova, $\mathrm{PhD}$ \\ The Republican Specialized Center of Cardiology, Tashkent, Uzbekistan
}

\begin{abstract}
Objective: to estimate the antihypertensive, antiremodelling efficacy and clinical tolerability of the monotherapy with Zofenopril in patients with Grade 1 and 2 hypertension (HT 1 and 2)

Materials and Methods: The study included 30 patients aged from 30 to 60 years with HT 1 and 2 (ESH/ESC, 2013) without severe comorbidities and cardiovascular complications. Zofenopril was prescribed as monotherapy to HT patients who had never been treated before or patients after one week of lavage from previous antihypertensive therapy, who did not reach target levels of blood pressure (BP). Before and during treatment all patients were checked on office BP using Korotkov's method and ambulatory blood pressure monitoring (ABPM). Echocardiography and Doppler sonography were carried out by standard methods using the recommendations of the American Society of Echocardiography. Intima-media thickness (IMT) of the carotid artery and brachial artery was measured by a $7.5 \mathrm{MHz}$ high-resolution ultrasound. Assessment of flow-mediated dilation (FMD) of the brachial artery was used as a method of determining endothelial function.

Results: A 12-week monotherapy with Zofenopril in average daily dose of 36.0 $9.54 \mathrm{mg}$ showed a high antihypertensive efficacy and a good safety profile without side effects. We noted a reliable decrease in systolic BP (SBP), diastolic BP (DBP), mean BP, and pulse pressure by $-19.53 \pm 5.93 \%,-18.64 \pm 7.18 \%,-19.05 \pm 6.14 \%$, and $-20.65 \pm 12.07 \%$, respectively. Target SBP, DBP, and SBP+DBP were reached in $90 \%, 86.6 \%$, and $83.3 \%$ of patients, respectively. We found a significant regression of LVH, significant improvement in volume indicators of LV echogeometry and parameters of FMD of the brachial artery, as well as a decrease in IMT of carotid and brachial arteries. Monotherapy with Zofenopril showed metabolic neutrality regarding the lipid and carbohydrate metabolism, a good safety profile without the side effects and undesired events. (Int J Biomed. 2015;5(4):198-202.)
\end{abstract}

Keywords: arterial hypertension; Zofenopril; antihypertensive efficacy; organprotective effects.

\section{Abbreviations}

LVEDV, left ventricular end-diastolic volume; LVESV, left ventricular end-systolic volume; LVM, left ventricular mass; LVH, left ventricular hypertrophy; RWT, relative wall thickness; IMT, intima-media thickness; FMD, flow-mediated dilation.

\section{Introduction}

For cardiology, the last quarter of the twentieth century was the era of angiotensin-converting enzyme (ACE) inhibitors. Captopril is the first representative of ACE inhibitors containing the sulfhydryl group. Over that period, many random clinical studies of Captopril were conducted,

*Corresponding author: Nigora Z. Srojidinova, PhD. The Republican Specialized Center of Cardiology, Tashkent, Uzbekistan. E-mail:nigora5505@gmail.com and it was shown to have high antihypertensive efficacy with cardio protection for patients with myocardial infarction and congestive heart failure, as well as nephroprotection for patients with diabetes and hypertension. Captopril had an advantage of organ protection over other ACE inhibitors because of the presence of the sulfhydryl group's antioxidant and anti-atherogenic features. However, Captopril started to give way to modern ACE inhibitors, mainly because of tissue affinity, lower compounding level with $\mathrm{ACE}$, and prolongation of dose, since a triple dose of Captopril is necessary to maintain daily effect. 
During the last 25 years, a new sulfhydryl ACE inhibitor, Zofenopril, was synthesized and clinically tested. This drug has many distinctive characteristics compared to its predecessor, Captopril. Zofenopril calcium is a highly lipophilic ACE inhibitor, which is converted to its active form, zofenoprilat, in both blood serum and various tissues. This is the essential difference between Zofenopril and other prodrugs such as Ramipril and Enalapril, which are largely activated only in blood serum and kidneys. Zofenopril, as other representatives of this class, inhibits ACE in plasma and tissues, as well as preventing degradation of bradykinin, which is known to be associated with cardio-vascular and renal effects of the drug. One of the main differences of Zofenopril is its high lipophilic level and affinity to ACE, which contribute to fast and full penetration and accumulation of the drug in the tissues and maximum inhibition of tissue ACE. Therefore, Zofenopril is associated with such pleiotropic effects as cardioprotection and prevention of endothelial dysfunction; anti-ischemic, antiinflammatory and antiatherogenic effects; its angiogenesis ability; and its ability to reverse development of apoptosis. The presence of the sulfhydryl group in Zofenopril gives it the ability to reduce oxidative stress, compound free radicals, increase production of nitric oxide, and provide an antiatherogenic and vasoprotective effect [1,2]. In accordance with that, Zofenopril increases coronary circulation and reduces ischemia, which lets us characterize Zofenopril as an antihypertensive drug with cardioprotective properties [3]. Organ-protective effects of antihypertensive drugs are especially necessary for HT treatment to achieve one of the main goals of antihypertensive therapy - prevention of targetorgan damage and reduction of cardiovascular risk.

Thereby, the aim of our study was to estimate the antihypertensive, antiremodelling efficacy and clinical tolerability of the monotherapy with Zofenopril in patients with Grade 1 and 2 hypertension (HT 1 and 2).

\section{Materials and Methods}

The study included 30 patients aged from 30 to 60 years (mean age 49.93 \pm 9.0 ) with HT 1 and 2 (ESH/ESC, 2013) [4] without severe comorbidities and cardiovascular complications (heart failure, cerebrovascular disease, myocardium infarction, diabetes mellitus). Zofenopril (Zocardis, Berlin-Chemi, MENARINI group) was prescribed as monotherapy to HT patients who had never been treated before or patients after one week of lavage from previous antihypertensive therapy, who did not reach target levels of blood pressure (BP). Before and during treatment all patients were checked on office BP using Korotkov's method and ambulatory blood pressure monitoring (ABPM) ("Registrator BR-102 plus" SCHILLER, Switzerland).

Echocardiography was carried out according to Penn Convention method [5] using «EnVisorC ()$_{\text {». The }}$ following parameters were measured and calculated: RWT, LVEDV, LVESV, EF, and LVM. LVM was indexed to body surface area (LVMI). Left ventricular hypertrophy (LVH) was defined as LVMI of $>95 \mathrm{~g} / \mathrm{m}^{2}$ (women) and $>115 \mathrm{~g} / \mathrm{m}^{2}$ (men) [4]. The diastolic function was evaluated by Doppler echocardiography. A decrease in PE/PA (peak early filling velocity/peak atrial filling velocity) ratio less than 1.0 was considered as a sign of diastolic dysfunction.

Intima-media thickness (IMT) of the carotid artery and brachial artery was measured by a $7.5 \mathrm{MHz}$ high-resolution ultrasound (EnVisorC $($ ). Assessment of flow-mediated dilation (FMD) of the brachial artery was used as a method of determining endothelial function [6]. The diameter of the brachial artery was measured from two dimensional ultrasound images, with the $7.5 \mathrm{MHz}$ linear array transducer. In each study, scans were taken at rest and during reactive hyperemia. FMD was estimated as the percent change in the diameter relative to the baseline diameter at rest. Level of FMD $\geq 10 \%$ was taken as the norm threshold [6].

The total cholesterol (TC), High-density lipoprotein cholesterol (HDL-C), and triglyceride (TG) levels were determined in the venous blood using the A-25 Biosystems Autoanalyzer (DAYTONA) and the "RENDOX" sets. Lowdensity lipoprotein cholesterol (LDL-C) was calculated according to Fridvald's formula. Fasting glucose and insulin were also determined.

After starting at $15 \mathrm{mg} /$ day, dosing was titrated to a maximum of $60 \mathrm{mg} /$ day at 4 -week intervals to achieve a target blood pressure of $<140 /<90 \mathrm{mmHg}$. The targeted lifestyle modifications were recommended for all patients. For patients with dyslipidemia, a lipid-lowering diet was also recommended.

The study was conducted in accordance with ethical principles of the Declaration of Helsinki. It was approved by the Republican Specialized Center of Cardiology Ethics Committee. Written informed consent was obtained from all participants.

Statistical analysis was performed using StatSoft Statistica v6.0 Baseline characteristics were summarized as frequencies and percentages for categorical variables and as $\operatorname{mean} \pm \mathrm{SD}$ for continuous variables. Student's unpaired and paired t-tests were used to compare two groups for data with normal distribution. Group comparisons with respect to categorical variables are performed using chi-square tests or, alternatively, Fisher's exact test when expected cell counts were less than 5. A probability value of $P<0.05$ was considered statistically significant.

\section{Results and Discussion}

Among studied patients, Grade 1 HT was identified in $46.7 \%$ of cases and Grade 2 HT in $53.3 \%$ of cases. Before therapy, systolic and diastolic BP were $149.67 \pm 9.99 \mathrm{mmHg}$ and $95.5 \pm 4.97 \mathrm{mmHg}$, respectively. HT duration was $5.07 \pm 4.38$ years. Fifty percent of patients suffered from first or second degree obesity (body mass index $>30 \mathrm{~kg} / \mathrm{m}^{2}$ ), only 5 patients had normal body weight and the rest were overweight. Overall, $60 \%$ of patients had LVH: $73.3 \%$ of cases for women, $53.3 \%$ for men. Dyslipidemia, thickening of IMT, impaired FMD, and fasting hyperglycemia were found in $70 \%, 56.6 \%, 53.3 \%$, and $16.6 \%$ of cases, respectively. Thus, more than half of the HT patients had a high cardiovascular risk. Average daily dose of Zofenopril was $36.0 \pm 19.54 \mathrm{mg}$, which was apportioned as 
follows: $10 / 33.3 \%$ patients took $15 \mathrm{mg} /$ day; $9 / 30 \%$ patients, $30 \mathrm{mg} /$ day; and $11 / 36.7 \%$ patients, $60 \mathrm{mg} /$ day.

A 12-week monotherapy with Zofenopril showed a high antihypertensive efficacy, which was evaluated by the BP decrease (more than 15\%) and achievement of target BP. We noted a reliable decrease in systolic BP (SBP), diastolic BP (DBP), mean BP (MBP), and pulse pressure (PP) by $-19.53 \pm 5.93 \%, \quad-18.64 \pm 7.18 \%, \quad-19.05 \pm 6.14 \%$, and $-20.65 \pm 12.07 \%$, respectively. Thus, target SBP, DBP, and SBP+DBP were reached in $90 \%, 86.6 \%$, and $83.3 \%$ of patients, respectively (Table 1).

Table 1.

BP indexes during 12-week monotherapy with Zofenopril

\begin{tabular}{|l|c|c|c|c|}
\hline \multicolumn{1}{|c|}{ Indexes } & SBP & DBP & MBP & PP \\
\hline $\begin{array}{l}\text { Before treatment, } \\
\text { mmHg }\end{array}$ & $\begin{array}{c}149.67 \\
\pm 9.99\end{array}$ & $\begin{array}{c}95.5 \\
\pm 4.97\end{array}$ & $\begin{array}{c}113.79 \\
\pm 6.34\end{array}$ & $\begin{array}{c}54.79 \\
\pm 6.44\end{array}$ \\
\hline After treatment, & $\begin{array}{c}120.33 \\
\pm 10.98 *\end{array}$ & $\begin{array}{c}77.67 \\
\pm 7.74 *\end{array}$ & $\begin{array}{c}91.89 \\
\pm 8.43 *\end{array}$ & $\begin{array}{c}42.67 \\
\pm 6.4 *\end{array}$ \\
mmHg & -19.53 & -18.64 & -19.05 & -20.65 \\
Level of BP & $\pm 5.93 \%$ & $\pm 7.18 \%$ & $\pm 6.14 \%$ & $\pm 12.07 \%$ \\
decrease, $\%$ & $90 \%$ & $86.6 \%$ & $83.3 \%$ & \\
\hline Target BP, $\%$ & &
\end{tabular}

$*_{-} P<0.0001$ - between before and after treatment

According to ABPM data, there was a significant reduction in average daily SBP and DBP and a tendency to reduction in average night systolic and diastolic BP indexes (Table 2).

\section{Table 2.}

ABPM indexes during 12-weekly monotherapy with Zofenopril

\begin{tabular}{|l|c|c|c|}
\hline \multicolumn{1}{|c|}{ Indexes } & Before treatment & $P$ & After treatment \\
\hline Average 24- h SBP, mmHg & $132.57 \pm 13.78$ & 0.036 & $128.07 \pm 13.23$ \\
\hline Average 24- h DBP, mmHg & $83.16 \pm 8.9$ & 0.047 & $79.7 \pm 8.26$ \\
\hline Daytime SBP, mmHg & $134.69 \pm 13.86$ & 0.041 & $129.8 \pm 12.86$ \\
\hline Daytime DBP, mmHg & $85.03 \pm 8.98$ & 0.07 & $81.63 \pm 8.62$ \\
\hline Nighttime SBP, mmHg & $125.39 \pm 15.33$ & 0.56 & $124.07 \pm 16.07$ \\
\hline Nighttime DBP, mmHg & $76.4 \pm 8.84$ & 0.4 & $75.07 \pm 10.05$ \\
\hline Nocturnal MBP fall,\% & $8.81 \pm 6.65$ & 0.34 & $9.07 \pm 6.93$ \\
\hline $\begin{array}{l}\text { Daytime SBP variability, } \\
\text { mm Hg }\end{array}$ & $14.09 \pm 3.11$ & 0.61 & $13.8 \pm 2.98$ \\
\hline $\begin{array}{l}\text { Daytime DBP variability, } \\
\text { mm Hg }\end{array}$ & $12.01 \pm 3.73$ & 0.55 & $11.58 \pm 2.49$ \\
\hline $\begin{array}{l}\text { Nighttime SBP variability, } \\
\text { mm Hg }\end{array}$ & $13.67 \pm 4.31$ & 0.03 & $11.59 \pm 2.75$ \\
\hline $\begin{array}{l}\text { Nighttime DBP variability, } \\
\text { mm Hg }\end{array}$ & $10.21 \pm 3.61$ & 0.27 & $9.31 \pm 2.45$ \\
\hline Daytime SBP load, \% & $34.36 \pm 28.15$ & 0.049 & $24.7 \pm 26.93$ \\
\hline Daytime DBP load, \% & $32.71 \pm 23.36$ & 0.054 & $23.69 \pm 23.02$ \\
\hline Nighttime SBP load, \% & $55.18 \pm 31.08$ & 0.64 & $52.04 \pm 32.66$ \\
\hline Nighttime DBP load, \% & $40.51 \pm 31.59$ & 0.13 & $30.76 \pm 31.15$ \\
\hline $\begin{array}{l}\text { RoR in morning SBP, } \\
\text { mmHg/h }\end{array}$ & $25.57 \pm 23.25$ & 0.39 & $22.13 \pm 19.19$ \\
\hline $\begin{array}{l}\text { RoR in morning DBP, } \\
\text { mmHg/h }\end{array}$ & $25.52 \pm 29.2$ & 0.57 & $20.74 \pm 21.72$ \\
\hline
\end{tabular}

The degree of nighttime BP reduction had a trend of decrease from $9.78 \pm 6.04$ to $7.77 \pm 8.23 \%$ during therapy $(\mathrm{P}=0.08)$. During the monotherapy, we noted a normalization of the degree of nighttime BP reduction in patients with significant abnormal nocturnal BP dipping status, which shows the safety of Zofenopril and the absence of night hypotension. Overall, to the end of observation, the normal degree of nocturnal BP fall was demonstrated in $50 \%$ of patients. Results showed that the other half of patients had impaired degrees of nocturnal BP fall during the monotherapy, which requires the addition of a second drug for these patients. During the 12-week therapy with Zofenopril, we noted a reduction in daytime SBP load and DBP load. Nighttime SBP load and DBP load also decreased, but without statistical reliability. Another significant indicator was the rate of rise (RoR) in BP in the morning, because the frequency of cardiovascular complications is greater during early morning hours. During therapy, RoR in SBP and DBP in the morning did not decrease enough: RoR for SBP and DBP was normalized in $22 \%$ and $40.7 \%$ of patients, respectively.

Reaching a target level of BP in more than $80 \%$ of patients proved the organ-protective effect of Zofenopril. We found a significant regression of $L V H$, significant improvement in volume indicators of LV echogeometry and parameters of FMD of the brachial artery, as well as a decrease in IMT of carotid and brachial arteries. Regression of LVH was evaluated by the dynamics of LVMM, LVMMI and RWT of LV (Table 3). A 12-week monotherapy with Zofenopril was associated with significant decrease in LVEDV and LVMI by $9.6 \pm 5.6 \%$. Zofenopril did not affect LV diastolic function.

Significant cardioprotective effects of Zofenopril were accompanied by expressed vasoprotection: the improvement and normalization in FMD of the brachial artery and positive dynamics in IMT of common carotid and brachial arteries (Table 3).

\section{Table 3.}

LV echogeometry and ultrasound vascular indexes during 12-week monotherapy with Zofenopril

\begin{tabular}{|l|c|c|c|}
\hline \multicolumn{1}{|c|}{ Indexes } & Before treatment & $P$ & After treatment \\
\hline LVEDV, $\mathrm{ml}$ & $140.08 \pm 29.24$ & 0.01 & $135.83 \pm 27.83$ \\
\hline LVESV, $\mathrm{ml}$ & $44.11 \pm 11.94$ & $N S$ & $43.6 \pm 11.23$ \\
\hline LVM, g & $231.14 \pm 73.5$ & 0.000 & $207.94 \pm 64.14$ \\
\hline LVMI, g/m ${ }^{2}$ & $117.84 \pm 32.5$ & 0.000 & $106.15 \pm 28.6$ \\
\hline PE/PA & $0.99 \pm 0.27$ & $N S$ & $0.97 \pm 0.26$ \\
\hline RWT, mM & $35.79 \pm 4.79$ & 0.000 & $34.25 \pm 4.61$ \\
\hline FMD, $\%$ & $9.86 \pm 3.82$ & 0.000 & $14.13 \pm 6.17$ \\
\hline IMTcc, mm & $1.0 \pm 0.3$ & 0.002 & $0.91 \pm 0.24$ \\
\hline IMTba, mm & $0.58 \pm 0.11$ & 0.005 & $0.56 \pm 0.09$ \\
\hline
\end{tabular}

IMTcc - IMT of common carotid artery; IMTba - IMT of brachial artery.

During 12-week monotherapy with Zofenopril, we did not reveal negative changes in the level of blood lipids and fasting blood glucose, which indicated the metabolic neutrality of Zofenopril (Table 4). A 12-week monotherapy with Zofenopril in average daily dose of $36 \pm 19.54 \mathrm{mg}$ was 
characterized with a good safety profile without side effects. All patients finished the 12-week study; compliance to therapy was high.

\section{Table 4.}

Blood lipids and fasting glucose during 12-week monotherapy with Zofenopril

\begin{tabular}{|l|c|c|c|}
\hline \multicolumn{1}{|c|}{ Indexes } & Before treatment & $P$ & After treatment \\
\hline TC, mg/dl & $234.13 \pm 45.87$ & $N S$ & $229.93 \pm 43.88$ \\
\hline TG, $\mathrm{mg} / \mathrm{dl}$ & $186.77 \pm 145.93$ & $N S$ & $178.7 \pm 138.02$ \\
\hline HDL, $\mathrm{mg} / \mathrm{dl}$ & $45.97 \pm 10.68$ & $N S$ & $45.4 \pm 10.96$ \\
\hline LDL, $\mathrm{mg} / \mathrm{dl}$ & $150.93 \pm 38.77$ & $N S$ & $143.2 \pm 35.9$ \\
\hline AI & $4.3 \pm 1.12$ & $N S$ & $4.35 \pm 1.46$ \\
\hline FG, $\mathrm{mmol} / 1$ & $5.21 \pm 0.62$ & $N S$ & $5.14 \pm 0.51$ \\
\hline
\end{tabular}

$A I$-atherogenic index; $F G$-fasting glucose.

\section{Discussion}

In many clinical studies, Zofenopril in a daily dose from 30 to $60 \mathrm{mg}$ showed high antihypertensive efficacy in patients with HT 1 and 2, which was not inferior when compared with Candesartan, Enalapril, Amlodipin, and Atenolol [7-9]. Many studies have demonstrated a smooth 24-hour antihypertensive effect of Zofenopril with normalization of daily BP profile. Decrease of BP in therapeutic diapason does not influence cerebral circulation, which remains on the necessary level even after reduced BP. Our research demonstrated a high antihypertensive efficacy, reaching the target level of BP in $83.3 \%$ of Grades 1 and 2 hypertensive patients. We revealed a significant normalization in daily, daytime and nighttime BP profile without the hypotonic episodes. The results obtained also showed the possibility of LVH regression, improvement and normalization of endothelial function, and reduction in IMT of the carotid and brachial arteries, which is associated with a positive effect on the prognosis of HT patients on the background of therapy with Zofenopril.

As is known, antecedent hypertension represents a risk factor for adverse outcomes in survivors of acute myocardial infarction (AMI). The results of SMILE study [10] suggest that treatment with Zofenopril started within $24 \mathrm{~h}$ of the onset of anterior AMI could be highly beneficial in patients with a history of HBP.

In SMILE-4 study, the efficacy of Zofenopril $60 \mathrm{mg}$ and acetylsalicylic acid (ASA) $100 \mathrm{mg}$ versus Ramipril $10 \mathrm{mg}$ and ASA was compared in patients with AMI complicated by left ventricular dysfunction, classified according to a history of hypertension. This retrospective analysis of the SMILE-4 study confirmed the good efficacy of Zofenopril and ASA in the prevention of long-term cardiovascular outcomes. The superiority of Zofenopril versus Ramipril was particularly evident in patients with isolated systolic hypertension $(\mathrm{n}=131$, $0.48(0.23-0.99), P=0.045)[11]$.

Zofenopril proved to be very effective in patients with coronary artery disease and myocardial infarction, thanks to its unique effective mechanism of action for improving blood pressure control, left ventricular function and myocardial ischemia burden, as well as angiotensin-converting enzyme inhibition. The SMILE project involved more than 3,500 patients with coronary artery disease and demonstrated that Zofenopril treatment may reduce mortality and morbidity in patients with myocardial infarction, also when combined with acetyl salicylic acid and to a greater extent than Lisinopril and Ramipril. In addition, the results of the SMILE-ISCHEMIA study have demonstrated an interesting anti-ischemic effect of Zofenopril, and these properties largely contribute to the overall clinical benefit of the drug. The effects of Zofenopril on blood pressure control and cardiovascular protection clearly support its primary role for prevention and treatment of cardiovascular diseases [12].

According to our results, we can draw some conclusions:

-The 12-week monotherapy with Zofenopril showed a high antihypertensive efficacy with a decrease in mean BP of $19 \%$ and achievement of the target BP in $83 \%$ patients with HT 1 and 2.

-According to ABPM, a full normalization of daily BP profile without the episodes of nightly hypotension was found in $50 \%$ of patients with HT 1 and 2 .

-Antiremodeling efficacy of a 12-week monotherapy with Zofenopril was characterized by LVH regression with LVMI reduction by $9.6 \%$, normalization in FMD of the brachial artery in $83.3 \%$ of patients, as well as a significant decrease in IMT of common carotid and brachial arteries.

-Monotherapy with Zofenopril showed metabolic neutrality regarding the lipid and carbohydrate metabolism, a good safety profile without the side effects and undesired events.

\section{Competing interests}

The authors declare that they have no competing interests.

\section{References}

1. Pasini AF, Garbin U, Nava MC. Stranieri C, Pellegrini M, Boccioletti V, et al. Effect of sulfhydryl and non-sulfhydryl angiotensin-converting enzyme inhibitors on endothelial function in essential hypertensive patients. Am J Hypertens. 2007; 20(4):443-50.

2. Evangelista S, Manzini S. Antioxidant and cardiopritective properties of the sulfhydryl angiotensin-converting enzyme inhibitor zofenopril. J Int Med Res. 2005; 33(1):42-54.

3. Ambrosioni E. Defining the role of zofenopril in the management of hypertension and ischemic heart disorders. Am J Cardiovasc Drugs. 2007; 7(1):17-24.

4. Mancia G, Fagard R, Narkiewicz K, Redon J, Zanchetti A, Böhm M, et al. $2013 \mathrm{ESH} / \mathrm{ESC}$ guidelines for the management of arterial hypertension: the Task Force for the Management of Arterial Hypertension of the European Society of Hypertension (ESH) and of the European Society of Cardiology (ESC). Eur Heart J. 2013;34(28):2159-219.

5. Devereux RB, Reichek N. Echocardiographic determination of left ventricular mass in man. Anatomic validation of the method. Circulation. 1977;55(4):613-8.

6. Celermajer DS, Sorensen KE, Gooch VM, Spiegelhalter DJ, Miller OI, Sullivan ID, et al. Non-invasive detection of endothelial dysfunction in children and adults at risk of 
atherosclerosis. Lancet.1992; 340(8828):1111-1115.

7. Nilsson P. Antihypertensive efficacy of zofenopril compared with atenolol in patients with mild to moderate hypertension. Blood Press Suppl. 2007;2:25-30.

8. Mallion JM. An evaluation of the initial and long-term antihypertensive efficacy of zofenopril compared with enalapril in mild to moderate hypertension. Blood Press Suppl. 2007;2:13-8.

9. Napoli C, Bruzzese G, Ignarro LJ, Crimi E, de Nigris $\mathrm{F}$, Williams-Ignarro $\mathrm{S}$, et al. Long-term treatment with sulfhydryl angiotensin-converting enzyme inhibition reduces carotid intima-media thickening and improves the nitric oxide/oxidative stress pathways in newly diagnosed patients with mild to moderate primary hypertension. Am Heart J. 2008;156(6):1154.e1-8.
10. Borghi C, Bacchelli S, Esposti DD, Bignamini A, Magnani B, Ambrosioni E. Effects of the administration of an angiotensin-converting enzyme inhibitor during the acute phase of myocardial infarction in patients with arterial hypertension. SMILE Study Investigators. Survival of Myocardial Infarction Long-term Evaluation. Am J Hypertens. 1999;12(7):665-72.

11. Borghi C, Ambrosioni E, Omboni S, Cicero AF, Bacchelli $\mathrm{S}$, Esposti DD,et al. Zofenopril and ramipril and acetylsalicylic acid in postmyocardial infarction patients with left ventricular systolic dysfunction: a retrospective analysis in hypertensive patients of the SMILE-4 Study. J Hypertens. 2013;31(6):1256-64. 12. 12. Borghi C1, Bacchelli S, Degli Esposti D. Long-term clinical experience with zofenopril. Expert Rev Cardiovasc Ther. 2012;10(8):973-82 